\title{
The effect of unilateral thalamic deep brain stimulation on the vocal dysfunction in a patient with spasmodic dysphonia: interrogating cerebellar and pallidal neural circuits
}

\author{
Anujan Poologaindran, BSc, ${ }^{1}$ Zurab Ivanishvili, MD, FRCSC, ${ }^{1}$ Murray D. Morrison, MD, FRCSC, ${ }^{2}$ \\ Linda A. Rammage, PhD, ${ }^{2}$ Mini K. Sandhu, RN, ${ }^{1}$ Nancy E. Polyhronopoulos, RN, BSN, ${ }^{1}$ and \\ Christopher R. Honey, MD, DPhil, FRCSC ${ }^{1}$
}

Department of Surgery, Divisions of ${ }^{1}$ Neurosurgery and ${ }^{2}$ Otolaryngology, The University of British Columbia, Vancouver, British Columbia, Canada

Spasmodic dysphonia (SD) is a neurological disorder of the voice where a patient's ability to speak is compromised due to involuntary contractions of the intrinsic laryngeal muscles. Since the 1980s, SD has been treated with botulinum toxin A (BTX) injections into the throat. This therapy is limited by the delayed-onset of benefits, wearing-off effects, and repeated injections required every 3 months. In a patient with essential tremor (ET) and coincident SD, the authors set out to quantify the effects of thalamic deep brain stimulation (DBS) on vocal function while investigating the underlying motor thalamic circuitry.

A 79-year-old right-handed woman with ET and coincident adductor SD was referred to our neurosurgical team. While primarily treating her limb tremor, the authors studied the effects of unilateral, thalamic DBS on vocal function using the Unified Spasmodic Dysphonia Rating Scale (USDRS) and voice-related quality of life (VRQOL). Since dystonia is increasingly being considered a multinodal network disorder, an anterior trajectory into the left thalamus was deliberately chosen such that the proximal contacts of the electrode were in the ventral oralis anterior (Voa) nucleus (pallidal outflow) and the distal contacts were in the ventral intermediate (Vim) nucleus (cerebellar outflow). In addition to assessing on/ off unilateral thalamic Vim stimulation on voice, the authors experimentally assessed low-voltage unilateral Vim, Voa, or multitarget stimulation in a prospective, randomized, doubled-blinded manner. The evaluators were experienced at rating SD and were familiar with the vocal tremor of ET. A Wilcoxon signed-rank test was used to study the pre- and posttreatment effect of DBS on voice.

Unilateral left thalamic Vim stimulation (DBS on) significantly improved SD vocal dysfunction compared with no stimulation (DBS off), as measured by the USDRS $(p<0.01)$ and VRQOL $(p<0.01)$. In the experimental interrogation, both low-voltage Vim $(p<0.01)$ and multitarget Vim + Voa $(p<0.01)$ stimulation were significantly superior to low-voltage Voa stimulation.

For the first time, the effects of high-frequency stimulation of different neural circuits in SD have been quantified. Unexpectedly, focused Voa (pallidal outflow) stimulation was inferior to Vim (cerebellar outflow) stimulation despite the classification of SD as a dystonia. While only a single case, scattered reports exist on the positive effects of thalamic DBS on dysphonia. A Phase 1 pilot trial (DEBUSSY; clinical trial no. NCT02558634, clinicaltrials.gov) is underway at the authors' center to evaluate the safety and preliminary efficacy of DBS in SD. The authors hope that this current report stimulates neurosurgeons to investigate this new indication for DBS.

https://thejns.org/doi/abs/10.3171/2016.10.JNS161025

KEY WORDS spasmodic dysphonia; deep brain stimulation; Vim; neuromodulation; focal; dystonia; functional neurosurgery

ABBREVIATIONS BTX = botulinum toxin A; DBS = deep brain stimulation; $\mathrm{ET}=$ essential tremor; $\mathrm{GPi}=$ internal globus pallidus; SD = spasmodic dysphonia; USDRS = Unified Spasmodic Dysphonia Rating Scale; Vim = ventral intermediate; Voa = ventral oralis anterior; VRQOL = voice-related quality of life.

SUBMITTED May 30, 2016. ACCEPTED October 31, 2016

INCLUDE WHEN CITING Published online March 17, 2017; DOI: 10.3171/2016.10.JNS161025 
$\mathrm{S}$ PASMODIC dysphonia (SD) is a neurological disorder of the voice where a patient's ability to speak is compromised due to involuntary contractions of the laryngeal muscles. ${ }^{3,5,25,50} \mathrm{SD}$ is the third most common focal dystonia,${ }^{25}$ following cervical dystonia and blepharospasm, and significantly limits an individual's ability to communicate effectively. Since the 1980s, the standard of care for SD has been to inject botulinum toxin A (BTX) into the laryngeal muscles. ${ }^{5}$ This treatment causes a chemical denervation, thereby diminishing the spasms. ${ }^{5}$ Unfortunately, BTX therapy has several limitations, rendering its use suboptimal. First, the clinical effect is temporary, and repeated injections are required approximately every 3 months. ${ }^{24}$ Second, there is a delay in the onset and a fading of the benefits, resulting in optimal symptom control for only a portion of a treatment cycle. ${ }^{24,31}$ Furthermore, some patients may develop neutralizing antibodies, rendering BTX ineffective. ${ }^{43}$ Another approach has been laryngeal denervation surgery. ${ }^{24}$ These approaches, mimicking the early treatments for cervical dystonia, ignore the central neurological dysfunction and focus on weakening the muscles so that the resulting spasms are diminished.

In adductor SD (80\%-90\% of SD cases), the vocal cords slam together and stiffen, making it difficult to produce speech. ${ }^{3}$ The thyroarytenoid muscle spasms force the vocal cords to adduct, producing a voice commonly described as strained, choppy, and full of effort. As a result, significant social embarrassment and inability to work and pursue hobbies can impact an affected individual's quality of life..$^{50}$ Very rarely, a patient with essential tremor (ET) requiring DBS may also have concurrent $\mathrm{SD}$. In one such patient with ET and coincident adductor SD, we investigated the effects of thalamic deep brain stimulation (DBS) on vocal function while interrogating the underlying pallidal and cerebellar circuitry.

\section{Case Report}

The University of British Columbia Clinical Research Ethics Board approved this study. The patient provided informed consent for participation in this study.

\section{Patient and Study Design}

A 79-year-old right-handed woman with ET was referred for left thalamic DBS surgery to ameliorate her dominant right upper limb tremor. This patient also had concurrent adductor SD that was being treated with BTX for the previous 2 years at our institution's voice clinic. A DBS electrode was placed into her left thalamic ventral intermediate (Vim) nucleus via a trajectory selected to place the more proximal contacts in her ventral oralis anterior (Voa) nucleus. Postoperatively, a mild change in the patient's speech was noted by the attending neurosurgeon. Six months after optimizing the stimulation to reduce her right upper-limb tremor, she entered the study. First, we compared measures of voice, and quality of life after 14 days of Vim DBS or 14 days of sham stimulation in a prospective, randomized, evaluator-blinded manner. Second, we prospectively evaluated her vocal characteristics after 5 days of randomized, double-blinded focal stimulation of the following nuclei: Vim, Voa, and Vim + Voa. Based on the fusion note, low voltages $(2.0 \mathrm{~V})$ in bipolar configuration were used in an attempt to focus the stimulation within the desired nuclei. Bipolar electrode settings were purposely chosen to narrow the electrical field and because the maximal current density is presumed to be near the cathodal (-) electrode contact.

\section{Surgery}

Standard frame-based stereotactic neurosurgical planning for Vim tremor surgery was conducted. We selected her left Vim target relative to the midcommissural point (MCP) to be anterior $-5.5 \mathrm{~mm}$, lateral $-12.6 \mathrm{~mm}$, and vertical $0.0 \mathrm{~mm}$ with an approaching ring angle of $60^{\circ}$ (arc angle of $18^{\circ}$ ). We deliberately selected this anterior approach so that the proximal contacts of the electrode would be in the Voa nucleus. We chose this in the mistaken belief that the proximal electrode contacts would benefit her SD, while her more distal electrode contacts in the Vim nucleus would benefit her tremor. Microelectrode recording of thalamic bursting phase locked with arm tremor and macrostimulation to block tremor was used to confirm electrophysiological placement of the DBS lead (Medtronic 3387).

\section{DBS Stimulation Parameters}

DBS stimulation parameters were initially programmed to maximally alleviate the patient's contralateral upper limb tremor, the original reason why this patient was referred to our team. We then evaluated her voice with the stimulation turned off for 14 days and on for 14 days. The left electrode's final stimulation parameters were: Case +, contacts, 0 off, 1-, 2 off, 3 off, pulse width $90 \mu \mathrm{sec}$, frequency $185 \mathrm{~Hz}$, and voltage $3.0 \mathrm{~V}$.

Since we had deliberately taken an anterior trajectory through the motor thalamus to experimentally assess the cerebellar and pallidal circuit's influence on voice, we were able to use low-voltage stimulation confined to each nuclei. Low-voltage focal Vim (cerebellar outflow) stimulation parameters were: contacts $0-, 1+, 60 \mu \mathrm{sec}, 185 \mathrm{~Hz}$, and $2.0 \mathrm{~V}$. Low-voltage focal Voa (pallidal outflow) stimulation parameters were: contacts $2-, 3+, 60 \mu \mathrm{sec}, 185 \mathrm{~Hz}$, and $2.0 \mathrm{~V}$. For multitarget stimulation, the stimulation parameters were: contacts $0-, 3+, 60 \mu \mathrm{sec}, 185 \mathrm{~Hz}$, and $2.0 \mathrm{~V}$.

\section{Voice Assessment}

The Unified Spasmodic Dysphonia Rating Scale (USDRS), ${ }^{44}$ a standardized voice scale to assess adductor SD severity, was completed independently by 2 raters. The raters, an experienced laryngologist (M.D.M.) and a speech language pathologist (L.A.R.), obtained a 95\% interrater reliability on sample recordings prior to evaluating study recordings. This evaluation was completed with the raters and patient blinded to the DBS settings. The patient, blinded to settings, also completed a voice-related quality of life (VRQOL) questionnaire, ${ }^{15}$ a widely accepted and standard questionnaire used in laryngology and speechlanguage pathology to assess QOL in dysphonic patients.

\section{Statisticical Analysis}

The patient in this study completed the USDRS and 
VRQOL assessments with DBS on (optimized for tremor control), off, and at the end of each experimental focal thalamic stimulation (Vim, Voa, and both) for a total of 5 trials. Statistical analysis of the resulting data was completed in 2 stages. Stage 1 involved comparing the USDRS and VRQOL subsections with DBS off versus on using a Wilcoxon signed-rank test. Stage 2 involved comparing different combinations of focal thalamic stimulation with each other as measured by the USDRS and VRQOL subsections using a Wilcoxon signed-rank test. Since calculating total USDRS and VRQOL would be statistically inappropriate for a single patient, we analyzed each of the USDRS's subsections after taking an average score between the raters for each of the 5 conditions. The subsections of the self-administered VRQOL were also analyzed in each of the 5 conditions. SPSS (version 22, IBM) was the statistical software used for interrater reliability and voice data analysis.

\section{Results}

Unilateral left thalamic Vim stimulation (DBS on) significantly improved SD vocal dysfunction compared with no stimulation (DBS off) as measured by the USDRS ( $p$ $<0.01)$ and VRQOL ( $p<0.01)$. Figure 1 depicts this improvement in vocal dysfunction as captured by the subsections of the USDRS and VRQOL. Voice recordings Audio 1 (DBS off) and Audio 2 (DBS on) highlight the striking improvement in the spasmodic dysphonic voice with DBS stimulation (audio content available online). Figure 2 is a postoperative CT scan with the Schaltenbrand-Wahren atlas overlay illustrating the location of each lead contact with respect to the individual thalamic nuclei. Contact 0 is in the ventral $\operatorname{Vim}(\mathrm{z}=0)$ and Contact 2 is in the Voa $(\mathrm{z}=$ 3 ). The Voa contact falls within a Voa-thalamotomy target of $1-5 \mathrm{~mm}$ above the AC-PC plane. ${ }^{16,46}$ Preoperatively, the right limb tremor was 38 as measured by the Fahn-TolosaMarin Tremor Rating Scale. ${ }^{10}$ During DBS on and DBS off, tremor was scored as 3 and 41, respectively.

In our experimental motor thalamic investigation, both low-voltage Vim $(\mathrm{p}<0.01)$ and multitarget Vim + Voa $(\mathrm{p}<$ 0.01 ) were significantly superior to low-voltage Voa stimulation as measured by the USDRS. There was no significant difference $(p>0.01)$ between low-voltage Vim and multitarget stimulation. Low-voltage Voa was ineffective, as Voa stimulation was worse than sham stimulation ( $p$ $<0.01$ ). Figure 3 is a boxplot comparison of voice during Vim, Voa, and multitarget thalamic stimulation as measured by the USDRS. A Wilcoxon-signed rank test was performed in SPSS to compare each of the settings, and $p$ values are provided in Fig. 3.

Qualitatively, a clinician not involved in the USDRS evaluation noted instantaneous improvement in dysphonia during the DBS on/off evaluations but also a 24-hour time period before full deterioration. This time course was interesting to us given the known DBS kinetics in ET (instantaneous) versus primary dystonia (weeks to months).

\section{Discussion}

The production of spoken language is a complex process that relies on multiple interacting brain regions, including the sensorimotor cortex, basal ganglia, thalamus, and cerebellum. ${ }^{39,40}$ When this neural network deviates from synchrony, speech disorders can occur, inducing voice tremor ${ }^{14}$ and SD. Due to its network-wide effects ${ }^{30}$ DBS represents an attractive therapeutic option to repair abnormal circuit dynamics in neurological speech disorders.

In this report, the effect of unilateral left thalamic DBS on the vocal dysfunction of SD has been quantified for the first time. While we report here only a single case, scattered reports exist on the benefits of thalamic DBS on the vocal dysfunction of SD. ${ }^{26,27,29,33}$ Marrying evidence from our work with these other studies, we hypothesize that the cerebellothalamic circuit may have an abnormal rhythm in patients with SD and that DBS may ameliorate this.

\section{Treating Cerebellar Dysfunction May Mediate Improvement in SD}

Most dystonias are currently treated with internal globus pallidus (GPi) DBS. ${ }^{48,49}$ A significant portion of the pallidal outflow is directed to the Voa thalamic nucleus. ${ }^{30}$ Some focal dystonias have been treated with thalamic lesions ${ }^{45}$ and neuromodulation of that portion of the motor thalamus. ${ }^{11}$ Since SD (also known as laryngeal dystonia) is a focal dystonia, we were surprised that Voa (pallidal outflow) thalamic stimulation produced no benefit in vocal dysfunction. It was clear that the best clinical effects for our patient's SD occurred with neuromodulation of the Vim (cerebellar outflow) thalamus.

Several recent lines of evidence point to cerebellar involvement in dystonia. Using the classic eye-blink conditioning paradigm, ${ }^{13}$ Teo and colleagues found physiological evidence for cerebellar dysfunction in patients with cervical and hand dystonia. ${ }^{47}$ The area of lobules $\mathrm{V}$ and VI of the cerebellar cortex have been shown to be structurally abnormal in patients with focal hand dystonia (writer's cramp) ${ }^{7}$ and cervical dystonia. ${ }^{9}$ Le Ber et al. described 8 dystonia-plus syndrome patients with predominant SD and cerebellar atrophy. ${ }^{22}$ Using PET imaging, Ali and colleagues demonstrated that therapeutic BTX injections decreased cerebellar hyperactivity in SD patients. ${ }^{1}$ Our recent study in a patient with hemidystonia demonstrated optimal clinical benefits when cerebellar circuits were included in neuromodulation. ${ }^{42}$ In a rodent study, Raike et al. used lentiviral-mediated conditional genetics to regionally limit cerebellar function. ${ }^{35}$ The authors found that abnormalities restricted to only $10 \%-15 \%$ of Purkinje cells were sufficient to induce focal dystonia. Koch et al. published a small study and reported that cerebellar stimulation improved cervical dystonia. ${ }^{21}$ Together, these studies provide evidence that cerebellar dysfunction is implicated in the genesis of focal dystonia and the production of speech.

Alcohol consumption is widely known to improve ET symptoms. PET studies in alcohol-responsive ET patients have demonstrated that alcohol reduces cerebellar hyperactivity. ${ }^{4}$ Recently, Kirke and colleagues determined that the symptoms improved with alcohol consumption in $60 \%$ of SD patients. ${ }^{20}$ Perhaps alcohol-induced suppression of SD works in a similar manner as tremor suppression in ET, providing additional support to treat the cerebellar dysfunction in SD. 


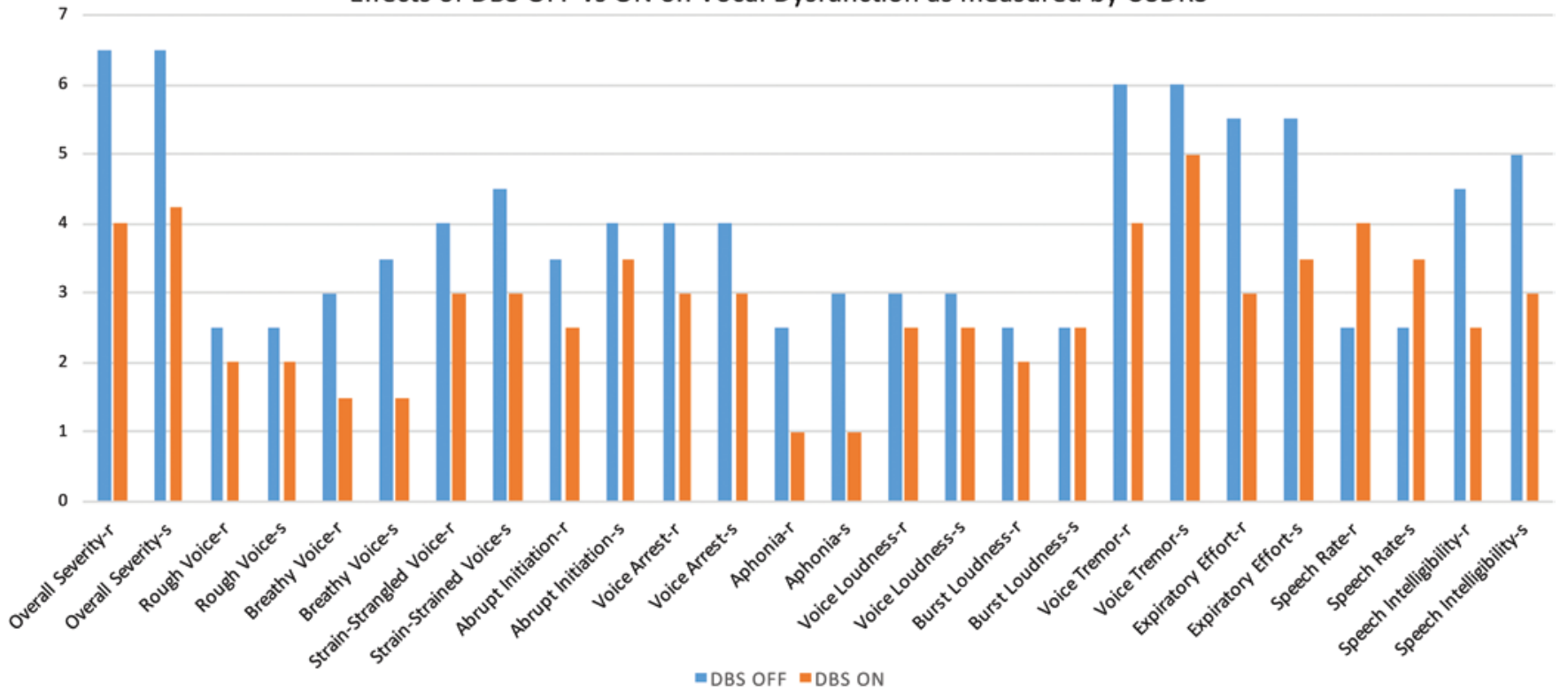

\section{Effects of DBS OFF vs ON on Vocal Dysfunction as Measured by VRQOL}

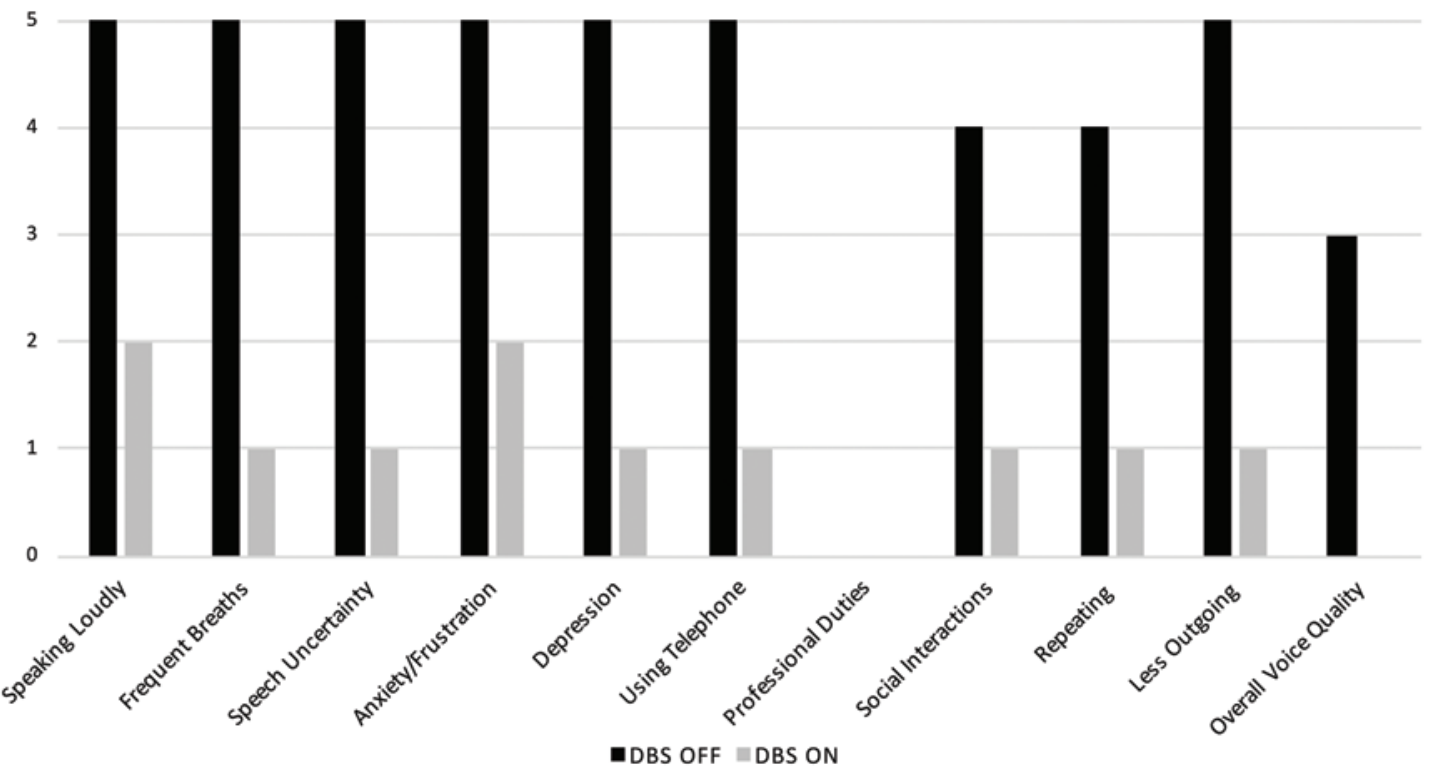

FIG. 1. Upper: DBS on (unilateral left Vim stimulation) compared with DBS off on the vocal dysfunction of SD as measured by the USDRS during reading $(r)$ and speaking (s) conditions. DBS on was significantly superior to DBS off $\left(p=1.11 \times 10^{-5}\right)$ on the Wilcoxon signed-rank test. Only Burst Loudness did not improve during the speaking state. An increase in speech rate on the USDRS represents an improvement. Lower: DBS on (unilateral left Vim stimulation) compared with DBS off on patient quality of life as measured by the VRQOL. DBS improved all aspects of quality of life and was significantly superior to DBS off $\left(p=3.99 \times 10^{-3}\right)$ on the Wilcoxon signed-rank test. Professional Duties was not applicable for this patient.

Finally, the clinical evidence following thalamic DBS for essential tremor and coincident SD is encouraging. ${ }^{26,27,29,33}$ Lyons et al. were the first to report that SD may respond to thalamic DBS. ${ }^{26,27}$ We have not yet tested the effect of GPi DBS on SD. Our data, together with that of other studies, hint that it may not be as effective as Vim DBS. Dystonia is increasingly being characterized as a multinodal network disorder where dysfunction at any single node can give rise to dystonia. ${ }^{18,34}$ Our initial impression is that SD requires targeting the Vim to maximally improve vocal dysfunction.

The coordination of speech production is facilitated by the cerebellar motor input to the laryngeal motor cortex via the motor thalamus.19,39,41 This neural circuit controls the 

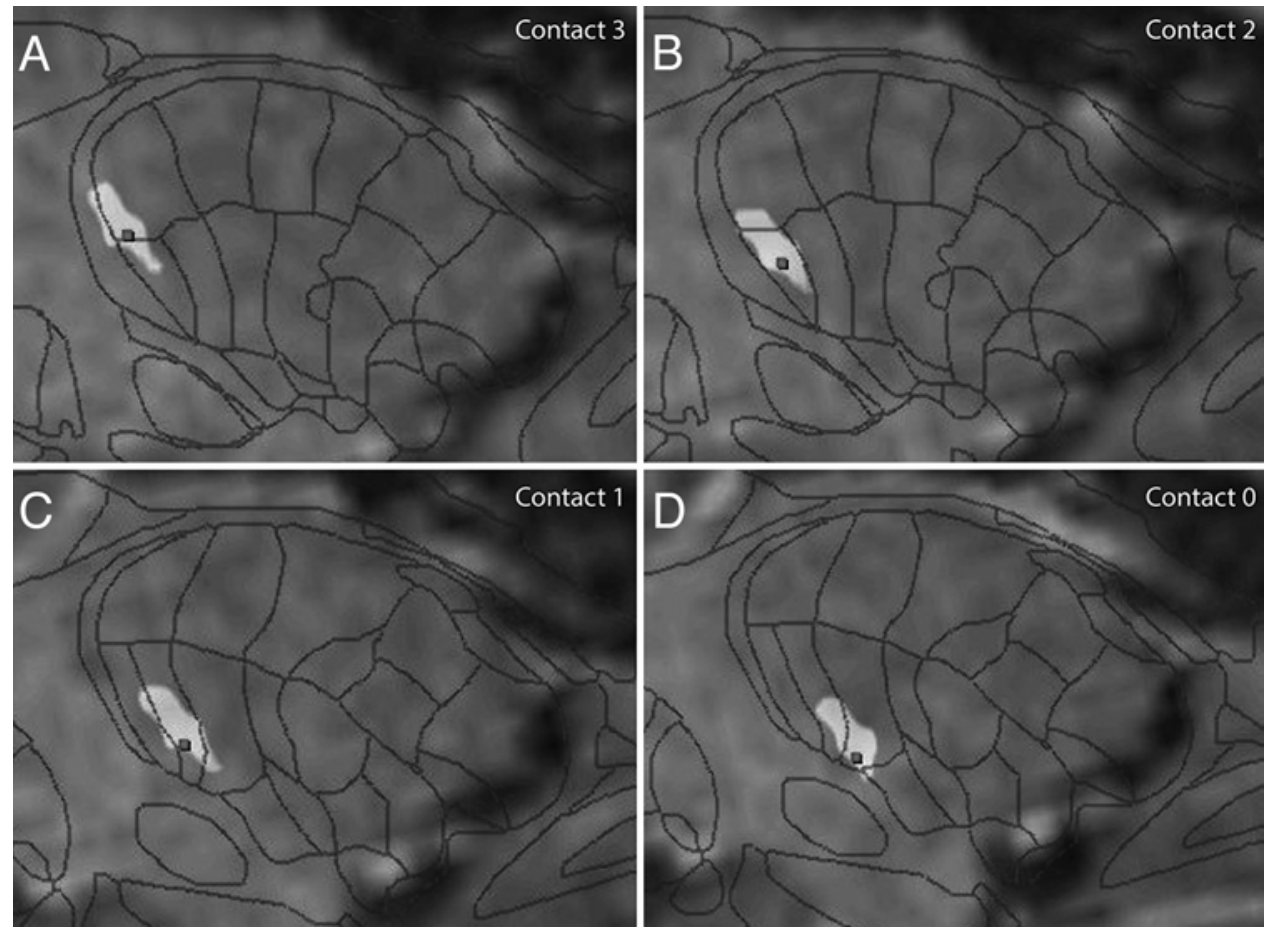

FIG. 2. Fusion of preoperative $M R I$ and postoperative $C T$ with Schaltenbrandt-Wahren atlas overlay illustrating the anterior DBS trajectory taken to place the left electrode. Contact $3(\mathbf{A})$ is in the anterosuperior border of Voa, Contact $2(B)$ is in the Voa, Contact 1 (C) is in the ventralis oralis posterior, and Contact 0 (D), the most distal, is in the Vim.

timing between single components of a movement, scales the size of muscular action, and coordinates the sequence of agonists and antagonists in normal speech production. ${ }^{8}$ While the basal ganglia undoubtedly plays an important role in limb, axial, and facial dystonia (including tongue), it appears that treating cerebellar dysfunction is required to correct abnormal speech coordination in SD.

\section{What About Pallidal Neuromodulation for SD?}

Contradictory reports exist on the value of GPi DBS in improving vocal dysfunction in SD and concurrent primary dystonia. Recently, Risch et al. reported on a single patient who had an "impressive" benefit in SD after GPi DBS for primary dystonia. ${ }^{38}$ Mure and colleagues reported on an SD and concurrent DYT6 dystonia in a patient who did not respond to GPi DBS but, interestingly, responded to thalamic ventral lateral anterior $\mathrm{DBS}^{29}$ - a pallidal receiving area of the thalamus. However, in a 2009 longterm outcomes report of GPi DBS for primary dystonia, the authors noted that of 10 patients with concurrent SD, 2 showed no benefit and 2 worsened after GPi DBS ${ }^{17}$ (unfortunately no information was provided for the remaining 6 patients). The authors noted that speech and swallowing were the only tested functions that did not improve at every time end point in their long-term pallidal DBS outcomes study. In another long-term follow-up study of 22 patients over 3 years, Vidailhet et al. also reported speech and swallowing as the only tested functions that did not significantly improve following GPi DBS. ${ }^{49}$ In a cohort of patients with Meige syndrome $(\mathrm{n}=7)^{36}$ and craniofacial/ cervical dystonia $(n=6),{ }^{23}$ pallidal DBS had no significant benefit on speech or swallowing. Thus, based on the available clinical evidence, pallidal neuromodulation has limited benefit on laryngeal dysfunction, whereas evidence for cerebellar neuromodulation has all been positive, especially in SD.

\section{Unilateral DBS for Spasmodic Dysphonia: Sufficient or Insufficient?}

Patel et al. reported subjective improvement in voice after unilateral left Vim DBS in a patient with ET who developed SD years after receiving DBS. ${ }^{33}$ Our patient also improved objectively after unilateral Vim DBS. Ali et al. suggested that the left hemisphere may play a cardinal role in adductor SD after determining asymmetrical hemispheric changes on PET following BTX injections. ${ }^{1}$ Moreover, a recent transcranial magnetic stimulation study found abnormal left motor cortex excitability during a "readingaloud" task in adductor SD patients compared with healthy controls; no significant changes in excitability were found in the right motor cortex..$^{45} \mathrm{~A}$ recent resting-state fMRI study found significant abnormal connectivity in the left inferior parietal cortex (together with the left sensorimotor cortex) in SD compared with healthy controls. ${ }^{2}$ This likely contributes to abnormal sensorimotor integration, loss of proprioceptive and tactile feedback, and inappropriate modulation of learned speech production present in SD. Finally, cerebellar stroke studies have implicated the right, not left, posterolateral cerebellum as critical for motor and cognitive aspects of speech. ${ }^{12,37}$ Together, these studies point toward a unifying theme: SD is a task-specific dystonia with neurophysiological speech motor control defects 


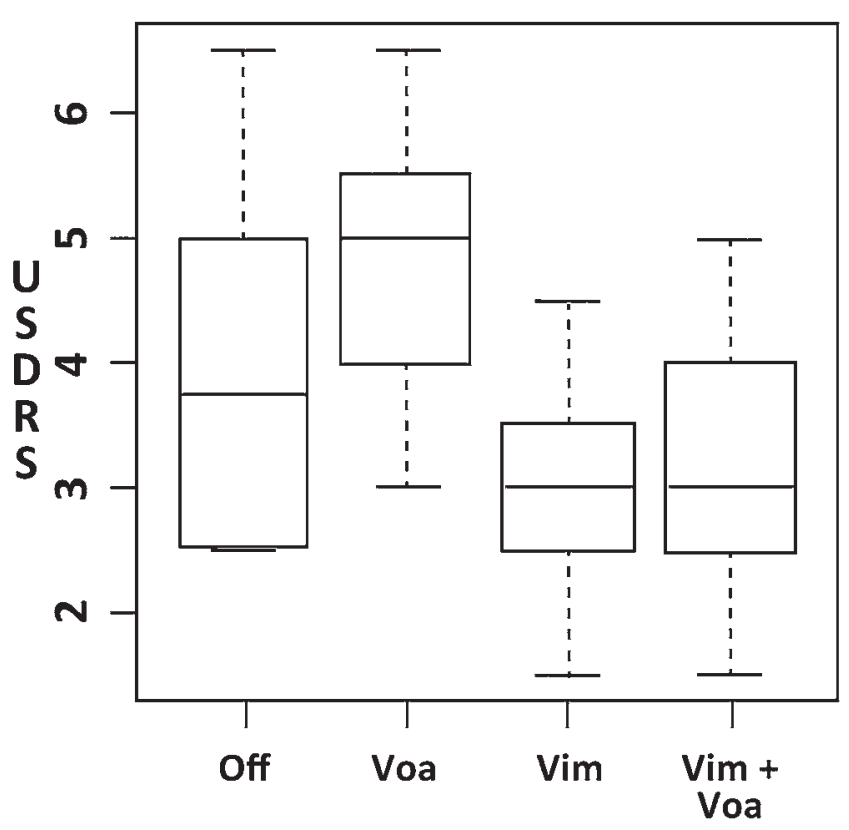

FIG. 3. Box-and-whisker plot comparing the effect of focal Vim, Voa, and Vim + Voa DBS stimulation on the vocal dysfunction of SD as measured by the USDRS (y-axis). The Wilcoxon signed-rank test was used with SPSS to compare each group. Statistical comparisons revealed the following: Voa focal stimulation was significantly worse than DBS off ( $p$ $=0.0028)$. Vim focal stimulation was significantly better than DBS off $(p=0.0016)$. Vim + Voa focal stimulation was significantly better than DBS off ( $p=0.0011$ ). Vim focal stimulation was significantly better than Voa focal stimulation $\left(p=2.69 \times 10^{-5}\right)$. Vim + Voa focal stimulation was significantly better than Voa focal stimulation $\left(p=2.05 \times 10^{-5}\right)$. There was no significant difference between Vim and Vim + Voa stimulation ( $p$ $=0.2003$ ). The box indicates the interquartile range; the line in the box, the median; and the whiskers, the full range.

primarily in the left cerebral hemisphere while relatively sparing the right hemisphere.

Ultimately, additional studies are needed to determine if unilateral or bilateral thalamic neuromodulation is required for optimal voice improvement in SD. Unilateral surgery has the obvious advantage of fewer brain penetrations, with a resultant reduction in complications. Our patient improved from unintelligible to easily understandable speech following a single DBS lead. Further clinically insignificant improvements in voice following contralateral surgery (if true) may not warrant the additional risks.

\section{Limitations of the Study}

An inherent limitation in the USDRS (and other adductor SD severity scales) is that the test is conducted under nonstressful conditions. Patients are asked to read standardized sentences in a quiet, isolated environment and are assessed on numerous vocal characteristics. ${ }^{44}$ In reality, our patient and others with SD reported that their symptoms are far worse under real-life stressful conditions, such as the workplace environment, public speaking, and socializing. The USDRS thus underestimates the SD symptom severity. In these real-life stressful conditions, our patient reported (subjectively) that the beneficial effects of DBS were even more profound.

The DBS settings were programmed to maximally al- leviate limb tremor. If patients with pure SD (no tremor) were to benefit from neuromodulation, programming parameters may be even better optimized to provide better voice benefits. Lastly, a small portion of the patient's vocal dysfunction was attributable to essential voice tremor. The improvement in her overall vocal quality of life (as measured by VRQOL) was therefore due to a reduction in both her SD and vocal tremor. Nevertheless, the coincidence of SD and voice tremor is around 50\%;, ,32 hence treating 2 disorders with 1 electrode is an exciting prospect that requires further research.

\section{Conclusions}

This report quantifies the beneficial effects of DBS on adductor SD while assessing the underlying thalamic circuitry for the first time. Unilateral neuromodulation of the left thalamus in the region of cerebellar input appears to be sufficient for clinically significant vocal improvement. Surprisingly, stimulating the pallidal receiving area of the thalamus (Voa) was ineffective and worse than sham stimulation. While our report is of only a single case, other reports exist on the positive effects of thalamic Vim DBS on dysphonia. Neuroimaging evidence also exists on cerebellar dysfunction in SD. Leaders in SD research have identified the need for a Phase 1 exploratory trial of DBS in patients with severe SD who are not adequately treated by BTX.25 A Phase 1 pilot trial (DEBUSSY, clinical trial no. NCT02558634, clinicaltrials.gov) is underway at our center to evaluate the safety and preliminary efficacy of DBS in SD.

\section{Acknowledgments}

We thank the Rare Disease Foundation and BC Children's Hospital Foundation for funding this project, Sherri Zelazny for her technical contributions, and Vivian Braithwaite for data collection. Most importantly, we thank our patient for her indomitable spirit in advancing medical research.

A.P., M.D.M., L.A.R., and C.R.H. (Principal Investigator) received a grant from The Rare Disease Foundation and BC Children's Hospital Foundation (\#19-2015) which supported this study. The sponsor had no role in the design or execution of the study.

\section{References}

1. Ali SO, Thomassen M, Schulz GM, Hosey LA, Varga M, Ludlow CL, et al: Alterations in CNS activity induced by botulinum toxin treatment in spasmodic dysphonia: an $\mathrm{H}_{2}{ }^{15} \mathrm{O}$ PET study. J Speech Lang Hear Res 49:1127-1146, 2006

2. Battistella G, Fuertinger S, Fleysher L, Ozelius LJ, Simonyan $\mathrm{K}$ : Cortical sensorimotor alterations classify clinical phenotype and putative genotype of spasmodic dysphonia. Eur J Neurol 23:1517-1527, 2016

3. Bielamowicz S, Stager SV, Badillo A, Godlewski A: Unilateral versus bilateral injections of botulinum toxin in patients with adductor spasmodic dysphonia. J Voice 16:117-123, 2002

4. Boecker H, Wills AJ, Ceballos-Baumann A, Samuel M, Thompson PD, Findley LJ, et al: The effect of ethanol on alcohol-responsive essential tremor: a positron emission tomography study. Ann Neurol 39:650-658, 1996

5. Brin MF, Blitzer A, Fahn S, Gould W, Lovelace RE: Adductor laryngeal dystonia (spastic dysphonia): treatment with local injections of botulinum toxin (Botox). Mov Disord 4:287-296, 1989 
6. Carpenter MA, Pahwa R, Miyawaki KL, Wilkinson SB, Searl JP, Koller WC: Reduction in voice tremor under thalamic stimulation. Neurology 50:796-798, 1998

7. Delmaire C, Vidailhet M, Elbaz A, Bourdain F, Bleton JP, Sangla S, et al: Structural abnormalities in the cerebellum and sensorimotor circuit in writer's cramp. Neurology 69:376-380, 2007

8. Diener HC, Dichgans J: Pathophysiology of cerebellar ataxia. Mov Disord 7:95-109, 1992

9. Draganski B, Thun-Hohenstein C, Bogdahn U, Winkler J, May A: "Motor circuit" gray matter changes in idiopathic cervical dystonia. Neurology 61:1228-1231, 2003

10. Fahn S, Tolosa E, Marin C: Clinical rating scale for tremor, in Jankovic J, Tolosa E (eds): Parkinson's Diseases and Movement Disorders, ed 2. Baltimore: Williams \& Wilkins, 1993

11. Fukaya C, Katayama Y, Kano T, Nagaoka T, Kobayashi K, Oshima H, et al: Thalamic deep brain stimulation for writer's cramp. J Neurosurg 107:977-982, 2007

12. Gebhart AL, Petersen SE, Thach WT: Role of the posterolateral cerebellum in language. Ann N Y Acad Sci 978:318333,2002

13. Gerwig M, Dimitrova A, Kolb FP, Maschke M, Brol B, Kunnel A, et al: Comparison of eyeblink conditioning in patients with superior and posterior inferior cerebellar lesions. Brain 126:71-94, 2003

14. Ho AL, Erickson-Direnzo E, Pendharkar AV, Sung CK, Halpern CH: Deep brain stimulation for vocal tremor: a comprehensive, multidisciplinary methodology. Neurosurg Focus 38(6):E6, 2015

15. Hogikyan ND, Sethuraman G: Validation of an instrument to measure voice-related quality of life (V-RQOL). J Voice 13:557-569, 1999

16. Horisawa S, Goto S, Nakajima T, Ochiai T, Kawamata T, Taira T: Stereotactic Thalamotomy for Hairdresser's Dystonia: A Case Series. Stereotact Funct Neurosurg 94:201-206, 2016

17. Isaias IU, Alterman RL, Tagliati M: Deep brain stimulation for primary generalized dystonia: long-term outcomes. Arch Neurol 66:465-470, 2009

18. Jinnah HA, Hess EJ: A new twist on the anatomy of dystonia: the basal ganglia and the cerebellum? Neurology 67:17401741,2006

19. Jürgens U: Neural pathways underlying vocal control. Neurosci Biobehav Rev 26:235-258, 2002

20. Kirke DN, Frucht SJ, Simonyan K: Alcohol responsiveness in laryngeal dystonia: a survey study. J Neurol 262:1548-1556, 2015

21. Koch G, Porcacchia P, Ponzo V, Carrillo F, Cáceres-Redondo MT, Brusa L, et al: Effects of two weeks of cerebellar theta burst stimulation in cervical dystonia patients. Brain Stimulat 7:564-572, 2014

22. Le Ber I, Clot F, Vercueil L, Camuzat A, Viémont M, Benamar N, et al: Predominant dystonia with marked cerebellar atrophy: a rare phenotype in familial dystonia. Neurology 67:1769-1773, 2006

23. Limotai N, Go C, Oyama G, Hwynn N, Zesiewicz T, Foote $\mathrm{K}$, et al: Mixed results for GPi-DBS in the treatment of cranio-facial and cranio-cervical dystonia symptoms. J Neurol 258:2069-2074, 2011

24. Ludlow CL: Treatment for spasmodic dysphonia: limitations of current approaches. Curr Opin Otolaryngol Head Neck Surg 17:160-165, 2009

25. Ludlow CL, Adler CH, Berke GS, Bielamowicz SA, Blitzer A, Bressman SB, et al: Research priorities in spasmodic dysphonia. Otolaryngol Head Neck Surg 139:495-505, 2008

26. Lyons MK, Adler CH, Bansberg SF, Evidente VGH: Spasmodic dysphonia may respond to bilateral thalamic deep brain stimulation. African J Neurol Sci 28:290, 2009
27. Lyons MK, Boucher OK, Evidente VGH: Spasmodic dysphonia and thalamic deep brain stimulation: long-term observations, possible neurophysiologic mechanism and comparison of unilateral versus bilateral stimulation. J Neurol Neurophysiol 1:106, 2010

28. Macchi G, Jones EG: Toward an agreement on terminology of nuclear and subnuclear divisions of the motor thalamus. $\mathbf{J}$ Neurosurg 86:77-92, 1997

29. Mure H, Morigaki R, Koizumi H, Okita S, Kawarai T, Miyamoto R, et al: Deep brain stimulation of the thalamic ventral lateral anterior nucleus for DYT6 dystonia. Stereotact Funct Neurosurg 92:393-396, 2014

30. Nakano K, Kayahara T, Tsutsumi T, Ushiro H: Neural circuits and functional organization of the striatum. J Neurol 247 (Suppl 5):V1-V15, 2000

31. Paniello RC, Barlow J, Serna JS: Longitudinal follow-up of adductor spasmodic dysphonia patients after botulinum toxin injection: quality of life results. Laryngoscope 118:564-568, 2008

32. Patel AB, Bansberg SF, Adler CH, Lott DG, Crujido L: The Mayo Clinic Arizona Spasmodic Dysphonia Experience: a demographic analysis of 718 patients. Ann Otol Rhinol Laryngol 124:859-863, 2015

33. Patel N, Richter A, Donovan D, Jimenez-Shahed J: Unilateral thalamic deep brain stimulation improves spasmodic dysphonia. Mov Disord 28 (Suppl 1):1231, 2013 (Abstract)

34. Prudente CN, Hess EJ, Jinnah HA: Dystonia as a network disorder: what is the role of the cerebellum? Neuroscience 260:23-35, 2014

35. Raike RS, Pizoli CE, Weisz C, van den Maagdenberg AM, Jinnah HA, Hess EJ: Limited regional cerebellar dysfunction induces focal dystonia in mice. Neurobiol Dis 49:200-210, 2013

36. Reese R, Gruber D, Schoenecker T, Bäzner H, Blahak C, Capelle HH, et al: Long-term clinical outcome in Meige syndrome treated with internal pallidum deep brain stimulation. Mov Disord 26:691-698, 2011

37. Richter S, Gerwig M, Aslan B, Wilhelm H, Schoch B, Dimitrova A, et al: Cognitive functions in patients with MRdefined chronic focal cerebellar lesions. J Neurol 254:11931203, 2007

38. Risch V, Staiger A, Ziegler W, Ott K, Schölderle T, Pelykh O, et al: How does GPi-DBS affect speech in primary dystonia? Brain Stimulat 8:875-880, 2015

39. Simonyan K: The laryngeal motor cortex: its organization and connectivity. Curr Opin Neurobiol 28:15-21, 2014

40. Simonyan K, Ludlow CL: Abnormal activation of the primary somatosensory cortex in spasmodic dysphonia: an fMRI study. Cereb Cortex 20:2749-2759, 2010

41. Simonyan K, Tovar-Moll F, Ostuni J, Hallett M, Kalasinsky VF, Lewin-Smith MR, et al: Focal white matter changes in spasmodic dysphonia: a combined diffusion tensor imaging and neuropathological study. Brain 131:447-459, 2008

42. Slotty PJ, Poologaindran A, Honey CR: A prospective, randomized, blinded assessment of multitarget thalamic and pallidal deep brain stimulation in a case of hemidystonia. Clin Neurol Neurosurg 138:16-19, 2015

43. Smith ME, Ford CN: Resistance to botulinum toxin injections for spasmodic dysphonia. Arch Otolaryngol Head Neck Surg 126:533-535, 2000

44. Stewart CF, Allen EL, Tureen P, Diamond BE, Blitzer A, Brin MF: Adductor spasmodic dysphonia: standard evaluation of symptoms and severity. J Voice 11:95-103, 1997

45. Suppa A, Marsili L, Giovannelli F, Di Stasio F, Rocchi L, Upadhyay N, et al: Abnormal motor cortex excitability during linguistic tasks in adductor-type spasmodic dysphonia. Eur J Neurosci 42:2051-2060, 2015

46. Taira T, Harashima S, Hori T: Neurosurgical treatment for writer's cramp. Acta Neurochir Suppl 87:129-131, 2003 
47. Teo JT, van de Warrenburg BP, Schneider SA, Rothwell JC, Bhatia KP: Neurophysiological evidence for cerebellar dysfunction in primary focal dystonia. J Neurol Neurosurg Psychiatry 80:80-83, 2009

48. Vidailhet M, Jutras MF, Grabli D, Roze E: Deep brain stimulation for dystonia. J Neurol Neurosurg Psychiatry 84:1029-1042, 2013

49. Vidailhet M, Vercueil L, Houeto JL, Krystkowiak P, Benabid AL, Cornu P, et al: Bilateral deep-brain stimulation of the globus pallidus in primary generalized dystonia. N Engl J Med 352:459-467, 2005

50. Zwirner P, Murry T, Swenson M, Woodson GE: Effects of botulinum toxin therapy in patients with adductor spasmodic dysphonia: acoustic, aerodynamic, and videoendoscopic findings. Laryngoscope 102:400-406, 1992

\section{Disclosures}

Dr. Honey reports that he receives non-study-related support for clinical or research effort from Medtronic, Boston Scientific, and St. Jude Medical.

\section{Author Contributions}

Conception and design: Honey. Acquisition of data: Poologaindran, Ivanishvili, Morrison, Rammage, Sandhu, Polyhronopoulos.
Analysis and interpretation of data: Poologaindran, Ivanishvili, Morrison, Rammage, Sandhu, Polyhronopoulos. Drafting the article: Poologaindran. Critically revising the article: Honey. Reviewed submitted version of manuscript: all authors. Approved the final version of the manuscript on behalf of all authors: Honey. Statistical analysis: Poologaindran. Study supervision: Honey.

\section{Supplemental Information}

Online-Only Content

Supplemental material is available with the online version of the article.

Audio 1 and Audio 2. https://thejns.org/doi/suppl/10.3171/2016. 10.JNS161025.

\section{Previous Presentations}

An abstract (oral presentation) of this work was presented at the XXII Congress of the European Society for Stereotactic and Functional Neurosurgery in Madrid, Spain, September 28-October 1, 2016.

\section{Correspondence}

Christopher R. Honey, Vancouver General Hospital, 8101-2775 Laurel St., Vancouver, BC V5Z 1M9, Canada. email: chris. honey@telus.net. 